\section{Ocean acidification science stands strong}

In his News Feature "Sea of doubts

( 7 May, p. 560), M. Enserink reports on fraud allegations in about one-fourth of the studies analyzing the impact of ocean acidification on fish behavior. As institutions work to determine whether there is truth to the allegations, which have not yet been independently verified, the public and policy-makers should remember that the outcome will not change the current scientific consensus: Ocean acidification is a major threat to marine species, ecosystems, and associated services.

No single article, research team, or even approach can explain the complexity of the consequences of ocean acidification (1). Over the past two decades, thousands of scientific articles have been published in this field, combining a wide range of approaches and methods from monitoring, paleo investigations, and modeling to laboratory, natural, and field experiments (2). The Fifth Assessment Report of the Intergovernmental Panel on Climate Change (IPCC) (3, 4) concluded with high confidence that both positive and negative impacts on marine organisms and ocean processes occur and that responses can be influenced, and often exacerbated, by other drivers such as warming and hypoxia. The effect on fish behavior is mentioned in the report, but it only marginally contributed to the IPCC conclusions and was assigned a medium to low confidence level in light of uncertainty on the long-term persistence of behavioral disturbances.

The science of ocean acidification stands strong whatever the outcome of the investigations of potential misconduct in the area of effects on fish behavior. Failure to quickly mitigate ocean acidification through ambitious reduction of $\mathrm{CO}_{2}$ emissions would have substantial consequences for the ocean and human societies.
Sam Dupont1*, Jean-Pierre Gat-

tuso $^{2}$, Hans-otto Pörtner ${ }^{3}$, Steve

Widdicombe 4

1University of Gothenburg, Fiskebäckskil, Sweden. 2Centre National de la Recherche Scientifique, Sorbonne Université, Iddri, France. ${ }^{3} \mathrm{Alfred}$ Wegener Institute, Bremerhaven, Germany. "Plymouth Marine Laboratory, Plymouth, UK. *Corresponding author. Email: sam.dupontegu.se

REFERENCES AND NOTES

1.B. Tilbrook et al., Front. Mar. Sci. 6, 337 (2019).

2.S. Doney et al., Annu. Rev. Environ. Resour. 45, 83 (2020).

3.J.-P.Gattuso et al., in Climate Change 2014: Impacts, Adaptation, and Vulnerability. Part A: Global and Sectoral Aspects. Contribution of Working Group II to the Fifth As sessment Report of the Intergovernmental Panel on Climate Change, C. B. Field et al., Eds. (Cambridge University Press, 2014), pp. 129-131.

4.H.-O. Pörtner et al., in Climate Change 2014: Impacts, Adaptation, and Vulnerability. Part A: Global and Sectoral Aspects. Contribution of Working Group II to the Fifth Assessment Report of the Intergovernmental Panel on Climate Change, C. B. Field et al., Eds., (Cambridge University Press, 2014), 411-484.

10.1126/science.abj4129 